\title{
Asian-American Acculturation, Severity of Concerns, and Willingness to See a Counselor
}

\author{
Ruth H. Gim, Donald R. Atkinson, and Scott Whiteley \\ University of California, Santa Barbara
}

\begin{abstract}
Asian Americans at a major West Coast university were surveyed to determine how severe each of 24 problems was for them and to assess their willingness to see a counselor for each problem. Factor analysis reduced the 24 problems to 8 areas of concern. A repeated measures analysis of variance revealed that severity ratings for the 8 concerns were related to acculturation and ethnicity. With severity ratings as covariates, a repeated measures analysis of covariance was computed for willingness to see a counselor ratings. Willingness to see a counselor was found to be related to both acculturation and gender across all 8 concerns. Women expressed greater willingness to see the counselor than did men and low-medium acculturated students expressed greater willingness to see a counselor than did high-acculturated students.
\end{abstract}

In an extensive review of research on counseling and psychotherapy with Asian Americans, Leong (1986) concluded that Asian Americans' underutilization of mental health services is well documented, despite some evidence of higher than normal need for services. A number of hypotheses, most of which assume a conflict between the psychotherapy process and the values of traditional Asian-American culture, have been offered to explain this pattern of underutilization. For example, it has been hypothesized that Asian Americans avoid mental health services because to seek outside help will bring shame upon their families (Root, 1985; Webster \& Fretz, 1978). It also has been hypothesized that Asian Americans underutilize mental health services because they are socialized to internalize stress and repress feelings (Sue \& Kitano, 1973; Sue \& Morishima, 1982). Furthermore, they may be socialized to believe that psychological problems are the result of bad thoughts and a lack of willpower and that they must resolve problems of this type on their own (Root, 1985).

Moreover, when Asian-American students do seek help, their presenting concerns are often different from Caucasian students. Sue and Kirk's (1975) study of students at the University of California, Berkeley, revealed that Asian-American students overutilized the campus counseling center but underutilized the psychiatric clinic when compared to general student use patterns. Sue and Kirk suggested that this was due to the less stigmatized services provided by the counseling center (e.g., academic and career counseling) than the services provided at the psychiatric clinic (e.g., mental health counseling); Asian Americans may be more reluctant than other groups to seek help for stigmatized mental health concerns. Tracey, Leong, and Glidden (1986) found additional support for this hypothesis. They reported that Asian-American clients were more likely to cite educational or vocational concerns

This study was made possible by a research grant from the Office of Vice Chancellor for Student Services at the University of California, Santa Barbara.

We thank Sharman L. Badget for her assistance in the completion of this survey.

Correspondence concerning this article should be addressed to Donald R. Atkinson, Graduate School of Education, University of California, Santa Barbara, California 93106. as their presenting issue whereas Caucasian clients were more likely to admit to personal or emotional concerns.

If Asian Americans' attitudes toward mental health services and their reluctance to use these services are a function of their basic cultural values, then it can be further hypothesized that more acculturated Asian Americans will be more willing to see a counselor than will less acculturated Asian Americans. An earlier study found some support for this hypothesis. Atkinson and Gim (1989) reported that Chinese, Japanese, and Korean Americans did not differ across ethnicity in their attitudes toward seeking professional psychological help but that more acculturated Asian Americans held more positive attitudes than did less acculturated Asian Americans. Other within-group differences among Asian Americans other than acculturation can be assumed to influence their attitudes toward mental health services. Although Atkinson and Gim found no gender or ethnic differences on attitudes toward seeking professional psychological help, Tracey et al. (1986) reported that female Chinese Americans and Filipino Americans were willing to identify more counseling-related concerns immediately before their intake interview than were their male counterparts.

The purposes of the current study were to examine: (a) the relation between ethnicity, gender, and acculturation and severity of problems experienced by Asian-American college students and (b) the relation between ethnicity, gender, and acculturation and willingness to see a counselor for problems experienced by Asian-American college students. We expected that less acculturated respondents would indicate more severe problems but would be less willing to see a counselor for these problems than would more acculturated respondents. Whereas we did not expect any ethnic group differences, we did anticipate that women would indicate a greater willingness to acknowledge problems and to see a counselor than would men.

\section{Method}

\section{Subjects}

The subjects for the study were 399 male and $\mathbf{4 1 7}$ female Asian American university students. Of these 816 respondents, 268 were 
Chinese Americans, 186 Filipino Americans, 151 Japanese Americans, 108 Korean Americans, and 103 Southeast-Asian Americans. Freshmen made up the largest group of subjects (291), followed by juniors (191), seniors (174), and sophomores (159), but 1 respondent did not indicate the year in college. The subjects' ages ranged from 16 to 37 years with a mean of 19.8 .

\section{Questionnaire}

The data for this study were collected as part of a larger survey that included an evaluation of student services available on the participating campus. For the purposes of this study, the questionnaire consisted of three parts: (a) a demographic section (gender, age, class standing, and ethnicity); (b) the Suinn-Lew Asian Self-Identity Acculturation scale (SL-ASIA); and (c) the modified Personal Problems Inventory (PPI).

The SL-ASIA (Suinn, Richard-Figueroa, Lew, \& Vigil, 1987) consists of 21 multiple-choice items that assess language preference (4 questions), identity (4 questions), friendship choice ( 4 questions), behaviors ( 5 questions), generation and geographic history ( 3 questions), and attitudes ( 1 question). Each item is rated on a scale of 1 (low acculturation) to 5 (high acculturation), and when all the items are summed, they generate a total score in a range from 21 to 105 . Suinn et al. referred to low, medium, and high scores on the SL-ASIA as, respectively, Asian identified, bicultural, and Western identified. They reported an alpha coefficient of .88 for the 21 items. The authors also used 3 individual items on the SL-ASIA as criteria for validating the overall instrument and reported a direct relation between scores on the SL-ASIA and (a) the generation since immigration of the respondent, (b) the length of residence in the United States of the respondent, and (c) self-ratings of cultural identity.

The PPI (Cash, Begley, McCown, \& Weise, 1975) consists of 15 problems identified by the authors as of concern to college students. This original list of 15 problems was expanded by Ponce and Atkinson (1989) to include five concerns that minority college students often encounter (adjustment to college, academic performance, financial concerns, feelings of loneliness and isolation, and feelings of alienation or not belonging). For the purposes of the current study, the list was further expanded to include four problems identified by a pilot study to be of particular concern to Asian-American students on the participating campus (trouble studying, ethnic or racial discrimination, roommate problems, ethnic identity confusion, general health problems). The participants in the current study were asked to make two ratings for each of the 24 problems. First, they were asked to rate the level of severity of each problem as it affected them on a 4-point scale $(1=$ not a problem to $4=$ major problem $)$; then they were asked to indicate their willingness to see a counselor for each problem on a 4 point scale $(1=$ not willing to $4=$ willing $)$.

\section{Procedure}

A total of 1,550 Asian-American students, the entire population of undergraduate students who identified themselves during fall-quarter registration as Asian Americans, who were enrolled at a major West Coast university were sent a battery of three questionnaires, one of which was the questionnaire for this study. Two mailings and a postcard reminder were conducted. Forty of the questionnaires were returned due to insufficient addresses, for a total of 1,510 possible respondents. Of these, 816 returned completed questionnaires for a usable response rate of $54 \%$.

\section{Results}

We factor analyzed student responses for the 24 ratings of problem severity in order to consolidate them into a smaller number of dependent variables (referred to hereafter as concerns). A principal-components factor analysis with a varimax rotation was used for this purpose. We selected a three-factor solution, following the discontinuity method; this solution accounted for $48 \%$ of the total variance, and the results of the factor analysis for students' severity ratings are reported in Table 1 . With a loading of .50 or above as the criterion for including an item in a factor, the three factors were identified as Relationship Concerns, Academic or Career Concerns, and Health or Substance Abuse Concerns. Within each of the three factors, the ratings on individual items that contributed to the factor were summed and then divided by the number of items that contributed to the total in order to create an average severity score for each of the three concerns. Five problems did not load over .50 on any factor and were treated as separate concerns. The three factored concerns and the five separate concerns served as dependent variables in subsequent analyses.

We followed Suinn et al.'s (1987) suggestion and initially divided the respondents into low, medium, and high levels of acculturation on the basis of their SL-ASIA scores (21-48 = low; $49-77=$ medium; and 78-105 = high). However, crosstabulation of acculturation level by gender and ethnicity revealed that there were no low-acculturated Japanese-American men and that there were low frequencies for other combinations of low acculturation. Therefore, acculturation was collapsed across low and medium scores to create two categories, low-medium and high.

A $2 \times 2 \times 5 \times 8$ repeated measures analysis of variance, with the main effects of respondent sex, level of acculturation, and ethnicity and with the eight concerns as the repeated

Table 1

Factor Loadings for Personal Concern Items

\begin{tabular}{lccc}
\hline \multicolumn{1}{c}{ Item } & Factor 1 & Factor 2 & Factor 3 \\
\hline General anxiety & .41293 & .52003 & .06424 \\
Alcohol problems & .01082 & .12029 & .79766 \\
Shyness & .71853 & .16414 & .09052 \\
College adjustment problems & .32638 & .53542 & .16358 \\
Sexual functioning problems & .50683 & .04779 & .32022 \\
Depression & .52926 & .41421 & .18464 \\
Conflicts with parents & .20239 & .32371 & .21794 \\
Academic performance & & & \\
problems & .10587 & .78111 & .07949 \\
Speech anxiety & .59495 & .22730 & .10653 \\
Dating or relationship problems & .62161 & .21808 & .05584 \\
Financial concerns & .16790 & .48087 & .25805 \\
Career choice problems & .11454 & .58832 & .18346 \\
Insomnia & .17543 & .35608 & .36517 \\
Drug addiction & .08453 & .07579 & .82227 \\
Loneliness or isolation & .71211 & .28784 & .21919 \\
Inferiority feelings & .69484 & .29769 & .13878 \\
Text anxiety & .29018 & .68983 & .01358 \\
Alienation & .76485 & .22857 & .21453 \\
Problems making friends & .78091 & .18959 & .14238 \\
Trouble studying & .17125 & .70432 & .10958 \\
Ethnic or racial discrimination & .52605 & .13037 & .41944 \\
Roommate problems & .35969 & .12227 & .35673 \\
Ethnic identity confusion & .36796 & .15600 & .45143 \\
General health problems & .14823 & .32393 & .56151 \\
\hline
\end{tabular}

Note. Factor loadings of items included in the three factored concerns (Relationship, Academic or Career, Health or Substance Abuse) are italicized. 
factors, was used to analyze the severity of concern ratings. As can be seen in Table 2, the main effects of acculturation, ethnicity, and concerns were all found to be significant. The interactions between acculturation and concerns and between ethnicity and concerns were also found to be significant.

The significant concerns effect indicates that severity ratings are a function of the type of concern rated. When collapsed across sex, acculturation, and ethnicity, the concern that received the highest severity rating was financial $(M=2.214)$, followed by academic or career $(M=2.122)$, relationship ( $M$ $=1.751)$, conflicts with parents $(M=1.683)$, ethnic identity confusion ( $M=1.469)$, roommate $(M=1.456)$, insomnia $(M=1.414)$, and health or substance abuse $(M=1.191)$ concerns. Tukey's honestly significance difference test revealed that the mean severity rating for financial concerns was significantly higher than all the other ratings, the mean severity rating for academic or career concerns was significantly higher than all but the financial concerns rating, the mean severity ratings for both conflicts with parents and relationship concerns were significantly higher than the mean ratings for ethnic identity confusion, roommate, insomnia, and health or substance abuse concerns, and the mean severity ratings for ethnic identity confusion, roommate, and insomnia concerns were significantly higher than the mean for health or substance abuse concerns. However, the significant Acculturation $\times$ Concerns and Ethnicity $\times$ Concerns interactions indicates that this order of severity ratings is not consistent across either the two levels of acculturation or the five ethnic groups. The source of the acculturation main effect and the acculturation by concerns interaction effect can be seen in Table 3. The acculturation main effect is accounted for by the consistently higher mean severity ratings given by the low-medium-acculturated students than by the high-acculturated students across all eight concerns. The Acculturation $\times$ Concerns interaction effect is apparently due to the different rankings of mean ratings within the two accultura-

Table 2

Repeated Measures Analysis of Variance for Severity of Concern Ratings

\begin{tabular}{|c|c|c|c|c|}
\hline Source & $S S$ & $d f$ & $M S$ & $F$ \\
\hline Mean & $7,741.035$ & 1 & $7,741.035$ & $4,960.53$ \\
\hline Gender (A) & 2.400 & l & 2.400 & 1.54 \\
\hline Acculturation (B) & 20.905 & 1 & 20.905 & $13.40^{* *}$ \\
\hline Ethnicity (C) & 21.944 & 4 & 5.486 & $3.52^{*}$ \\
\hline$A \times B$ & 5.523 & 1 & 5.523 & 3.54 \\
\hline$A \times C$ & 7.005 & 4 & 1.751 & 1.12 \\
\hline$B \times C$ & 3.962 & 4 & 0.991 & 0.63 \\
\hline $\mathrm{A} \times \mathrm{B} \times \mathrm{C}$ & 4.015 & 4 & 1.004 & 0.64 \\
\hline Error & $1,178.197$ & 755 & 1.561 & \\
\hline Concerns (D) & 279.368 & 7 & 39.910 & $96.01^{* * *}$ \\
\hline$D \times A$ & 5.827 & 7 & 0.832 & 2.00 \\
\hline $\bar{D} \times \mathrm{B}$ & 14.365 & 7 & 2.052 & $4.94^{* * *}$ \\
\hline $\mathrm{D} \times \mathrm{C}$ & 33.963 & 28 & 1.213 & $2.92^{* * *}$ \\
\hline$D \times A \times B$ & 3.955 & 7 & 0.565 & 1.36 \\
\hline $\mathrm{D} \times \mathrm{A} \times \mathrm{C}$ & 11.901 & 28 & 0.425 & 1.02 \\
\hline $\mathrm{D} \times \mathrm{B} \times \mathrm{C}$ & 9.184 & 28 & 0.328 & 0.79 \\
\hline $\mathrm{D} \times \mathrm{A} \times \mathrm{B} \times \mathrm{C}$ & 11.586 & 28 & 0.414 & 1.00 \\
\hline Error & $2,196.868$ & 5285 & 0.416 & \\
\hline
\end{tabular}

Note. The Geisser-Greenhouse conservative test was applied to all $F$ values that involved the repeated factor.

${ }^{*} p<.01 . \quad{ }^{* *} p<.001 . \quad{ }^{* * *} p<.0001$.
Table 3

Mean Ratings for Severity of Concern by Acculturation Level

\begin{tabular}{|c|c|c|c|c|}
\hline \multirow[b]{3}{*}{ Concerns } & \multicolumn{4}{|c|}{ Level of acculturation } \\
\hline & \multicolumn{2}{|c|}{ Low-medium } & \multicolumn{2}{|c|}{ High } \\
\hline & $M$ & $S D$ & $M$ & $S D$ \\
\hline Relationship & 1.859 & 0.733 & 1.575 & 0.645 \\
\hline Academic or career & 2.228 & 0.722 & 1.949 & 0.635 \\
\hline Health or substance abuse & 1.218 & 0.528 & 1.146 & 0.399 \\
\hline Conflicts with parents & 1.766 & 0.924 & 1.548 & 0.732 \\
\hline Financial & 2.415 & 1.055 & 1.884 & 0.931 \\
\hline Insomnia & 1.456 & 0.732 & 1.344 & 0.716 \\
\hline Roommate & 1.504 & 0.790 & 1.378 & 0.723 \\
\hline Ethnic identity confusion & 1.504 & 0.777 & 1.412 & 0.664 \\
\hline
\end{tabular}

tion groups. Low-medium-acculturated students gave their highest mean severity rating to financial problems and second highest mean rating to academic or career problems; this order was reversed for high-acculturated students.

The source of the ethnicity main effect can be traced in Table 4 to the consistency with which Southeast Asian Americans gave the highest problem severity ratings across all the concerns (with one exception, conflicts with parents) and Japanese Americans gave the lowest ratings (with one exception, health or substance abuse problems). The Ethnicity $x$ Problems interaction effect can be explained by ranking the mean severity ratings within ethnic groups. Chinese and Japanese Americans gave their highest severity ratings to academic or career problems and their second highest ratings to financial problems; this order was reversed for Korean, Filipino, and Southeast Asian Americans.

The willingness to see a counselor ratings for the 24 problems also were consolidated to reflect the eight concerns established through the factor analysis of severity ratings. Scores for the willingness to see a counselor for relationship, academic or career, and health or substance abuse concerns were averaged to create an identical 1-7 score range for all eight dependent variables. The eight willingness to see a counselor ratings were then analyzed by means of $2 \times 2 \times 5$ $\times 8$ repeated measures analysis of covariance, with main effects of respondent sex, level of acculturation, ethnicity, and concerns and with the eight severity ratings as the covariates. This analysis, reported in Table 5 , resulted in significant main effects for gender, acculturation, and concerns; all other main and interaction effects were nonsignificant. The significant concerns effect indicates that willingness to see a counselor ratings are a function of the type of concern rated. The highest adjusted mean willingness to see a counselor rating was recorded for academic or career $(M=1.394)$ concerns followed by financial ( $M=1.344)$, conflicts with parents $(M=1.073)$, relationship $(M=1.068)$, ethnic identity confusion $(M=$ $1.010)$, roommate $(M=0.993)$, health or substance abuse ( $M$ $=0.991)$, and insomnia $(M=0.945)$ concerns. Tukey's honestly significant difference test revealed that the mean adjusted willingness to see a counselor rating for financial concerns was significantly higher than all the other ratings, the mean willingness rating for academic or career concerns was significantly higher than for all but the financial concerns rating, and the mean willingness ratings for both parental conflict and relationship concerns were significantly higher 
Table 4

Mean Ratings for Severity of Concern by Ethnicity

\begin{tabular}{lcccccc}
\hline & \multicolumn{5}{c}{ Ethnicity } \\
\cline { 2 - 6 } \multicolumn{1}{c}{ Concerns } & Chinese & Japanese & Korean & Filipino & $\begin{array}{c}\text { Southeast } \\
\text { Asian }\end{array}$ \\
\hline Relationship & 1.785 & 1.571 & 1.629 & 1.746 & 2.083 \\
Academic or career & 2.064 & 1.905 & 2.057 & 2.299 & 2.354 \\
Health or substance abuse & 1.147 & 1.156 & 1.152 & 1.209 & 1.365 \\
Conflicts with parents & 1.749 & 1.435 & 1.714 & 1.751 & 1.729 \\
Financial & 2.044 & 1.830 & 2.210 & 2.401 & 2.906 \\
Insomnia & 1.367 & 1.354 & 1.476 & 1.401 & 1.583 \\
Roommate & 1.466 & 1.354 & 1.381 & 1.480 & 1.625 \\
Ethnic identity confusion & 1.478 & 1.340 & 1.476 & 1.435 & 1.698 \\
\hline
\end{tabular}

than the mean insomnia rating. The significant gender effect was due to consistently higher adjusted mean willingness to see a counselor ratings for women than for men. The significant acculturation effect was due to consistently higher adjusted means for low-medium-acculturated students than for high-acculturated students.

\section{Discussion}

The results of this study indicate that acculturation, gender, and ethnicity are all related to the types of concerns that Asian Americans experience and their willingness to see a counselor for these concerns. Acculturation and ethnicity were found to be related to the severity of concerns experienced by Asian-American students and acculturation and gender were found to be related to willingness to see a counselor about those concerns.

With respect to severity of concerns, the results of this study suggest that Asian Americans perceive financial and academic

Table 5

Repeated Measures Analysis of Covariance for Willingness to See Counselor Ratings

\begin{tabular}{|c|c|c|c|c|}
\hline Source & $S S$ & $d f$ & $M S$ & $F$ \\
\hline Gender (A) & 9.045 & 1 & 9.045 & $6.36^{*}$ \\
\hline Acculturation (B) & 7.538 & 1 & 7.538 & $5.30^{*}$ \\
\hline Ethnicity (C) & 7.183 & 4 & 1.796 & 1.26 \\
\hline $\mathrm{A} \times \mathrm{B}$ & 0.060 & 1 & 0.060 & 0.04 \\
\hline$A \times C$ & 7.517 & 4 & 1.879 & 1.32 \\
\hline $\mathrm{B} \times \mathrm{C}$ & 2.840 & 4 & 0.710 & 0.50 \\
\hline$A \times B \times C$ & 3.887 & 4 & 0.972 & 0.68 \\
\hline Covariate & $1,622.175$ & 1 & $1,622.175$ & $1,140.59$ \\
\hline Error & $1,006.936$ & 708 & 1.422 & \\
\hline Concerns (D) & 68.650 & 7 & 9.807 & $22.46^{* *}$ \\
\hline$D \times A$ & 5.427 & 7 & 0.775 & 1.78 \\
\hline $\mathbf{D} \times \mathbf{B}$ & 2.563 & 7 & 0.366 & 0.84 \\
\hline $\mathrm{D} \times \mathrm{C}$ & 11.030 & 28 & 0.394 & 0.90 \\
\hline$D \times A \times B$ & 2.828 & 7 & 0.404 & 0.92 \\
\hline $\mathrm{D} \times \mathrm{A} \times \mathrm{C}$ & 8.438 & 28 & 0.301 & 0.69 \\
\hline $\mathrm{D} \times \mathrm{B} \times \mathrm{C}$ & 7.018 & 28 & 0.251 & 0.57 \\
\hline$D \times A \times B C$ & 5.753 & 28 & 0.205 & 0.47 \\
\hline Covariate & $2,885.320$ & 1 & $2,885.320$ & $6,606,49$ \\
\hline Error & $2,167.104$ & 4962 & 0.437 & \\
\hline
\end{tabular}

Note. The Geisser-Greenhouse conservative test was applied to all $F$ values that involved the repeated factor.

${ }^{*} p<.05 .{ }^{* *} p<.0001$. or career concerns as their greatest problems. Some evidence was found, however, that low-medium-acculturated Asian Americans rate financial concerns as a greater problem than academic or career concerns, whereas their high-acculturated counterparts reversed this order. Both acculturation groups gave their lowest severity rating to health or substance abuse concerns. The results also suggest that acculturation may be inversely related to the severity of concerns experienced by Asian Americans. This may be because less acculturated Asian Americans experience more psychosocial stressors than their more acculturated counterparts. It can be argued that less acculturated Asian Americans experience a greater degree of conflict between the Asian and American cultures, whereas the more acculturated Asian Americans have generally adopted the values, norms, and lifestyle of the majority culture and therefore may not experience as much cultural conflict.

Language fluency also may account for the higher severity ratings by low-medium-acculturated respondents. Because length of residence in the U.S. is directly related to level of acculturation, we can infer that less acculturated Asian Americans have been in the U.S. for a shorter period of time than more acculturated Asian Americans and thus are less likely to be fluent in English. This, in turn, may generate stress in a variety of areas. Southeast Asian Americans may be particularly affected because most of them have entered the U.S. since 1974.

Contrary to our expectation, the results of this study suggest that ethnicity may be related to the severity of psychological concerns experienced by Asian Americans. Southeast Asian Americans in this study expressed the greatest concern across seven of the eight problem areas, whereas Japanese Americans expressed the least concern for seven of the eight problem areas. However, these findings must be viewed with some caution, given the unequal cell frequencies for the crosstabulation of ethnicity by acculturation. The ratio of lowmedium-acculturated to high-acculturated respondents was almost 12 to 1 for Southeast Asian Americans, whereas for Japanese Americans it was 1 to 2.6. Thus, the ethnicity main effect may be due largely to a disproportionate number of Southeast Asian Americans who were low-medium acculturated and to a disproportionate number of Japanese Americans who were high acculturated.

At the same time, the effects of war on immigrants of Southeast Asia could help explain the greater concern they 
experience for most of the problems. In Ruth H. Gim's experience working with Southeast Asian students on academic probation, some of these students have described symptomatology characteristic of posttraumatic stress disorder, including difficulty in concentrating, feelings of depression for no apparent reason, and recurring nightmares (of the violent deaths of loved ones or personal experience with torture). Posttraumatic stress disorder may explain in part why Southeast Asian Americans seem to report greater concern for various types of problems than do other Asian Americans.

With respect to willingness to see a counselor, our results indicate that Asian Americans, in general, are most willing to see a counselor about academic or career and financial concerns and least willing to see a counselor about ethnic identity confusion, roommate, health or substance abuse, and insomnia concerns. The fact that Asian-American college students in this study gave both their highest severity ratings and their highest willingness to see a counselor ratings to financial, academic or career, relationship, and conflicts with parents concerns suggests that counseling centers ought to be prepared to address these problems with their Asian-American clients. The fact that Asian Americans gave their lowest severity and willingness to see a counselor ratings to insomnia, health or substance abuse, roommate, and ethnic identity confusion concerns, all four of which fall within the purview and expertise of university counseling services, suggests that counseling centers may need to promote their image in these areas among Asian Americans.

Acculturation and gender also were found to be related to respondents' willingness to see a counselor for the eight concerns. Contrary to our expectation, however, an inverse relation was found between acculturation and willingness to see a counselor. This appears to conflict with Atkinson and Gim's (1989) findings that more acculturated subjects were more likely than less acculturated subjects to recognize a personal need for professional psychological help, to be tolerant of the stigma associated with psychological help, and to be open to discussing their problems with a psychologist. In our study the respondents first rated how severe each concern was for them, and this rating served as a covariate for an analysis of their willingness to see a counselor for a number of specific problems. It may be that when less acculturated students do acknowledge that they have a concern, they are more willing than more acculturated students to seek professional help with the concern. This, in turn, may reflect a greater respect for authority on the part of less acculturated Asian Americans than on the part of more acculturated Asian Americans.

Women were found to be more willing than men to see a counselor across all eight concerns examined. This tends to support a similar finding by Tracey et al. (1986) that AsianAmerican women were more willing to endorse counseling- related problems than men and may be reflective of earlier findings that women in the general student population are more willing to seek professional psychological help than are men (Fischer \& Turner, 1970; Williams, 1983).

This study is subject to the limitations of any survey study. Furthermore, the respondents in this study were college students, and the results may not be generalizable to noncollege populations. To the degree these results are generalizable, however, they suggest that acculturation is an important within-group variable that may relate to severity of psychological problems and willingness to seek help for those problems among Asian Americans.

\section{References}

Atkinson, D. R., \& Gim, R. H. (1989). Asian-American cultural identity and attitudes toward mental health services. Journal of Counseling Psychology, 36, 209-212.

Cash, T. F., Begley, P. J., McCown, D. A., \& Weise, B. C. (1975). When counselors are heard but not seen: Initial impact of physical attractiveness. Journal of Counseling Psychology, 22, 273-279.

Fischer, E. H., \& Turner, J. L. (1970). Orientations to seeking help: Development and research utility of an attitude scale. Journal of Consulting and Clinical Psychology, 35, 79-90.

Leong, F. T. L. (1986). Counseling and psychotherapy with AsianAmericans: Review of the literature. Journal of Counseling Psychology, 33, 196-206.

Ponce, F. Q., \& Atkinson, D. R., (1989). Mexican-American acculturation, counselor ethnicity, counseling style, and perceived counselor credibility. Journal of Counseling Psychology, 36, 203-208.

Root, M. P. P. (1985). Guidelines for facilitating therapy with Asian American clients. Psychotherapy, 22, 349-356.

Sue, S., \& Kitano, H. H. (1973). Stereotypes as a measure of success. Journal of Social Issues, 29, 83-98.

Sue, D. W., \& Kirk, B. A. (1975). Asian-Americans: Use of counseling and psychiatric services on a college campus. Journal of Counseling Psychology, 22, 84-86.

Sue, S., \& Morishima, J. K. (1982). The mental health of AsianAmericans. San Francisco: Jossey-Bass.

Suinn, R. M., Richard-Figueroa, K., Lew, S., \& Vigil, P. (1987). The Suinn-Lew Asian Self-Identity Acculturation Scale: An initial report. Educational and Psychological Measurement, 47, 401-407.

Tracey, T. J., Leong, F. T. L., \& Glidden, C. (1986). Help seeking and problem perception among Asian Americans. Journal of Counseling Psychology, 33, 331-336.

Webster, D. W., \& Fretz, B. R. (1978). Asian American, black, and white college student's preferences for help-giving services. Journal of Counseling Psychology, 25, 124-130.

Williams, J. H. (1983). Psychology of women (2nd ed.). New York: Norton.

Received July 28, 1989

Revision received November 6, 1989

Accepted December 19, 1989 